\title{
Cataract Surgery as a Risk Factor for Retinal Detachment in Very Highly Myopic
}

Eyes

Guido Ripandelli, MD,${ }^{1}$ Cecilia Scassa, MD ${ }^{1}$ Vincenzo Parisi, MD,${ }^{1,2}$ Daniela Gazzaniga, MD ${ }^{1}$ Donald J. D'Amico, MD, ${ }^{3}$ Mario Stirpe, $M D^{1}$

Purpose: To evaluate the incidence of retinal detachment (RD) after cataract surgery performed by phacoemulsification in very highly myopic eyes.

Design: Retrospective, paired-eye, case-control trial.

Participants and Intervention: We assessed the development of RD in 930 eyes from 930 subjects (mean age $=62.5 \pm 8.5$ years) affected by very high myopia (between -15 and -30 diopters) undergoing cataract surgery after uncomplicated phacoemulsification (cataract-subjected [CS] eyes). Fellow eyes served as controls. Follow-up was 36 months.

Main Outcome Measure: Detachment of the retina.

Results: Retinal detachment was observed in $8.0 \%$ of CS eyes compared with $1.2 \%$ of control eyes $(P<0.01$, chi-square test). In CS eyes, posterior RD was most common (52.7\% of eyes with RD). In control eyes, peripheral detachments with or without macular involvement were most common (47.3\% of eyes with RD).

Conclusion: Cataract surgery, despite the use of a safe technique such as phacoemulsification, increases the risk of RD development in very highly myopic eyes. Ophthalmology 2003;110:2355-2361 () 2003 by the American Academy of Ophthalmology.

Cataract extraction has been considered a risk factor for retinal detachment (RD) in highly myopic eyes. ${ }^{1-4}$ It has been reported that the incidence of RD is different when emmetropic and myopic eyes are compared. ${ }^{5-7}$ In addition, a varying incidence of RD has been observed in relation to the degree of myopia. ${ }^{7-11}$

Several studies have suggested that the introduction of new surgical techniques may reduce the incidence of $\mathrm{RD}$ after cataract extraction. ${ }^{12-16}$

The aim of our study was to evaluate the incidence of RD after cataract surgery performed by phacoemulsification in highly myopic eyes.

\footnotetext{
Originally received: November 11, 2001.

Accepted: March 27, 2003.

Manuscript no. 210598.

${ }^{1}$ Fondazione G. B. Bietti per l'Oftalmologia, Rome, Italy.

${ }^{2}$ Cattedra di Clinica Oculistica, Universita' di Roma "Tor Vergata," Rome, Italy.

${ }^{3}$ Massachusetts Eye and Ear Infirmary, Harvard Medical School, Boston, Massachusetts.

Presented in part at the annual meeting of the American Academy of Ophthalmology, New Orleans, Louisiana, November 2001.

The study protocol was designed by one of the authors (MS) in association with Ronald G. Michels, MD (deceased). With the conclusion of the study, the authors honor the memory of Dr Michels.

Each author states that he has no proprietary interest in the development or marketing of the instruments used.

Correspondence to Guido Ripandelli, MD, Via Pasubio 6, 00195 Roma, Italy. E-mail: vparisi@tin.it.
}

\section{Methods}

\section{Subjects}

Between January 1991 and December 1997, we enrolled 1024 highly myopic phakic subjects who underwent uneventful cataract surgery in one eye only. The degree of myopia observed in these 1024 subjects before surgical treatment (see below) ranged from -15 to -30 diopters (D), with a mean of $-20.8 \pm 4.3 \mathrm{D}$. This group included 499 men and 525 women, and the age of the subjects ranged from 39 to 81 years (mean age $=62.6 \pm 8.8$ years). In addition to having an uncomplicated procedure, inclusion criteria were as follows:

- no previous history of eye surgery, glaucoma, or ocular trauma;

- no presence of Wagner's or Stickler's syndrome;

- intraocular pressure of $<20 \mathrm{mmHg}$;

- no previous history of diabetes or other systemic disease that might confound the visual acuity; and

- a difference in the degree of myopia not exceeding $\pm 2 \mathrm{D}$ between the eye undergoing cataract surgery and the fellow eye that served as the control eye (CE).

Control eyes were all phakic and were not subjected to cataract surgery during the 36-month period of follow-up (see below).

Informed consent was obtained from each subject enrolled in the study, and the research followed the tenets of the Declaration of Helsinki.

In baseline conditions (preoperatively), the following examinations were performed on both eyes of all subjects enrolled in the study:

- best-corrected visual acuity assessed by Snellen table, 
Table 1. Characteristics of the 930 Myopic Subjects Subjected to Cataract Surgery

\begin{tabular}{|c|c|c|c|c|c|c|c|c|c|c|c|c|}
\hline \multirow[b]{3}{*}{ Group } & \multirow[b]{3}{*}{$\mathrm{N}$} & \multirow{2}{*}{\multicolumn{2}{|c|}{ Gender }} & \multirow[b]{3}{*}{ Age (yrs) } & \multirow{2}{*}{\multicolumn{2}{|c|}{$\begin{array}{l}\text { Degree of Myopia } \\
\text { (negative diopters) }\end{array}$}} & \multirow{2}{*}{\multicolumn{2}{|c|}{$\begin{array}{c}\text { Posterior } \\
\text { Staphyloma } \\
\end{array}$}} & \multicolumn{4}{|c|}{ Baseline Retinal Degeneration } \\
\hline & & & & & & & & & \multicolumn{2}{|c|}{ CS Eyes } & \multicolumn{2}{|c|}{ CEs } \\
\hline & & $M$ & $F$ & & CS Eyes & CEs & CS Eyes & $\overline{C E s}$ & Treated & Untreated & Treated & Untreated \\
\hline A & 431 & 207 & 224 & $61.5 \pm 8.2$ & $15-19$ & $15-19$ & 48 & 36 & 188 & 38 & 143 & 42 \\
\hline B & 372 & 158 & 214 & $60.3 \pm 9.3$ & $20-24$ & $20-24$ & 166 & 179 & 152 & 29 & 119 & 31 \\
\hline C & 127 & 62 & 65 & $63.3 \pm 7.8$ & $25-30$ & $25-30$ & 127 & 127 & 46 & 19 & 36 & 25 \\
\hline Total & 930 & 427 & 503 & $62.5 \pm 8.5$ & $20.7 \pm 4.2$ & $21.0 \pm 3.8$ & 341 & 342 & 386 & 86 & 298 & 98 \\
\hline
\end{tabular}

$\mathrm{N}=$ number of patients; $\pm=$ one mean standard deviation; $\mathrm{CS}=$ cataract-subjected; $\mathrm{CE}=$ control eyes.

- dilated indirect binocular ophthalmoscopy with scleral depression,

- slit-lamp examination supplemented with a +90-D lens,

- fundus photography, and

- ultrasonographic A-scan and B-scan examinations by means of a $10-\mathrm{MHz}$ probe on an A/B scan system (Humphrey Instruments, San Leandro, CA).

In eyes subjected to cataract surgery and in fellow eyes, which served as CEs, all these examinations were performed postoperatively at $1,3,6,9,12,18,24,30$, and 36 months after cataract surgery performed by phacoemulsification.

During the first six-month period of follow-up, 94 subjects retired from the study. The most common reason was the discomfort created by the unilateral modification of refraction after cataract surgery. In addition, some subjects from other cities were put off by the inconvenience of traveling for all the follow-up visits. The 94 retired subjects did not differ from the remaining 930 subjects regarding mean degree of myopia, mean age, gender distribution, and the clinical findings of the preoperative fundus eye examinations.

We, thus, considered only subjects who had followed all complete postoperative examinations at the preestablished times. Ultimately, 930 cataract-subject (CS) eyes and the relative $930 \mathrm{CEs}$ were included in the study and considered in the statistical evaluation (see below).

The degree of myopia observed in these 930 subjects before surgical treatment ranged from -15 to $-30 \mathrm{D}$, with a mean of $-20.7 \pm 4.2 \mathrm{D}$. This group included 427 men and 503 women, and the age of the subjects ranged from 39 to 81 years (mean age $=$ $62.5 \pm 8.5$ years).

The details of subjects included in this study are summarized in Table 1.

At baseline conditions, consistent vitreous modifications characterized by structural changes such as posterior vitreous detachment (PVD) or extensive vitreous liquefaction and posterior vitreous schisis were found in 894 subjects. Prophylactic laser treatment was carried out in $386(41.5 \%)$ of the 930 CS eyes and in $298(32.0 \%)$ of the $930 \mathrm{CEs}$. Of the 386 eyes subjected to cataract surgery, 346 had scars of laser treatment around areas of the lattice (148 eyes), snail tracks ( 80 eyes), symptomatic (30 eyes) or asymptomatic (53 eyes) retinal holes, and symptomatic ( 9 eyes) or asymptomatic (26 eyes) tears; 40 eyes had $360^{\circ}$ peripheral scars of laser treatment without evidence of areas of retinal dystrophy. Of the 930 CEs, 263 had scars of laser treatment around areas of the lattice (97 eyes), snail tracks (63 eyes), symptomatic ( 21 eyes) or asymptomatic (48 eyes) retinal holes, and symptomatic (12 eyes) or asymptomatic ( 22 eyes) tears; 35 eyes had $360^{\circ}$ peripheral scars of laser treatment without evidence of areas of retinal dystrophy. Untreated, minor peripheral areas of lattice degeneration were found in $86 \mathrm{CS}$ eyes and in 98 CEs.

The presence of a posterior staphyloma, revealed by ultrasono- graphic B-scan examination, was observed in 342 of 930 CEs $(36.8 \%)$ and in 341 of 930 CS eyes (36.7\%).

The 930 eyes subjected to cataract surgery were divided into three groups on the basis of the degree of myopia and axial length as assessed by ultrasonographic B-scan examination:

- group A: 431 eyes with myopia between -15 and -19 D (axial length between 29.70 and $31.26 \mathrm{~mm}$ );

- group B: 372 eyes with myopia between -20 and -24 D (axial length between 31.65 and $33.21 \mathrm{~mm}$ ); and

- group C: 127 eyes with myopia between -25 and $-30 \mathrm{D}$ (axial length between 33.60 and $35.55 \mathrm{~mm}$ ).

\section{Surgical Treatment}

Briefly, retrobulbar or peribulbar anesthesia was used in all eyes. Cataract surgery was performed using a frown-shaped, self-sealing, superior scleral tunnel with an incision of 3 to $5 \mathrm{~mm}$. Capsulorrhexis was performed as well as four-quadrant cracking phacoemulsification. The posterior capsule was routinely polished, and a posterior one-piece intraocular polymethyl methacrylate lens (IOL) of $5.5 \mathrm{~mm}$ in diameter was implanted in the capsular bag of 387 of the 930 eyes. The surgery was, by the study design and inclusion criteria, uncomplicated in all eyes. Postoperatively, subjects received an antibiotic-steroid medication. During the 36month period of follow-up, yttrium-aluminum-garnet (YAG) laser capsulotomy was performed on 318 of the 930 (34.2\%) cataract surgery-treated eyes.

\section{Statistics}

The differences observed between the group of CS eyes and the group of CEs have been evaluated by two-by-two tables and chi-square tests, assuming that both eyes of individual subjects may contribute independently to the observed incidence results. Given the high number of comparisons, a conservative $P$ value $<$ 0.01 was adopted as statistically significant.

\section{Results}

The main results presented in Table 2 show the development of $\mathrm{RD}$ in CS eyes and CEs during the 36-month period of follow-up.

During the 36-month period of follow-up, the development of RD was observed in 11 of 930 CEs $(1.2 \%)$, and we found the development of RD in 74 of $930 \mathrm{CS}$ eyes $(8.0 \%, P<0.001$ vs. CEs).

In CEs, RD occurred within the first 12 months in two eyes (18.2\%), between 13 and 24 months of follow-up in four eyes (36.4\%), and between 25 and 36 months of follow-up in five eyes $(45.5 \%)$. 
Ripandelli et al · Cataract Surgery as a Risk Factor for Retinal Detachment

Table 2. Development of Retinal Detachment (RD) in Myopic Subjects during the 36-month Period of Follow-up

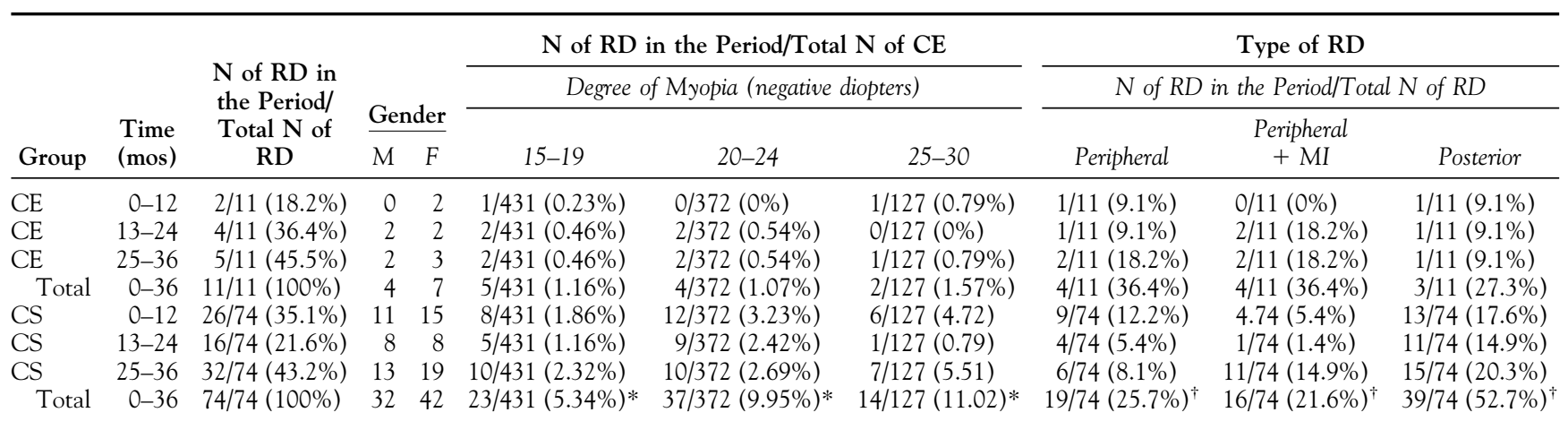

$\mathrm{N}=$ number; $\mathrm{CE}=$ control eye; $\mathrm{CS}=$ cataract-subjected; $\mathrm{MI}=$ macular involvement.

$* P<0.01$, chi-square with respect to CEs.

${ }^{\dagger} P>0.01$, chi-square with respect to CEs.

In CS eyes, RD occurred within the first 12 months after surgery in 26 eyes $(35.1 \%, P>0.05$ vs. CEs), between 13 and 24 months after surgery in 16 eyes $(21.6 \%, P>0.05$ vs. CEs), and between 25 and 36 months after surgery in 32 eyes $(43.2 \%, P>$ 0.05 vs. CEs).

There was no significant difference $(P>0.05)$ in mean age or gender in the development of RD during the 36-month period of follow-up.

We observed three types of RD, which we defined as "peripheral" (RD originating from peripheral holes, tears, vitreoretinal tractions, or degenerative areas and extending no further than the postequatorial region), "peripheral with macular involvement" (RD with the same peripheral origin and clinical features as mentioned above but involving the macular region), and "posterior" (RD originating in the posterior pole from a macular hole, paravascular tears, and sometimes from tangential posterior tractions without evidence of retinal breaks).

\section{Control Eyes}

In CEs, although the majority of cases of RD belonged to group A (eyes with myopia ranging from -15 to $-19 \mathrm{D}, 5$ of $431 \mathrm{CS}$ eyes with RD [1.16\%]), considering the number of eyes in each group, a larger incidence of RD ( 2 of $127 \mathrm{CS}$ eyes with RD [1.57\%]) was observed in group C (eyes with myopia ranging from -25 to -30 D).

The type of RD according to degree of myopia is described in Table 3

In CEs, peripheral detachment with or without macular involvement was more common $(72.7 \%)$ than posterior RD $(27.3 \%)$.

Peripheral RD was found in 4 CEs $(36.3 \%)$, peripheral RD with macular involvement was found in 4 CEs $(36.3 \%)$, and posterior RD was observed in 3 CEs $(27.3 \%)$.

No posterior staphyloma was detected in the 4 CEs with peripheral RD; the presence of a posterior staphyloma was observed in 1 of the $4(25 \%)$ CEs with peripheral RD plus macular involvement and in 2 of the $3(66.7 \%)$ CEs with posterior RD. Details are presented in Table 4.

In the eight $\mathrm{CE}$ eyes with peripheral RD, there was PVD. RD was generally severe and originated from superior retinal breaks on areas of lattice degeneration in 2 of the 8 eyes and from retinal tears or holes in the inferior quadrants in the other 6 eyes. In all of the three eyes with PVD, there was vitreous liquefaction with a posterior vitreous schisis. In all cases, RD, which was mainly flat, originated from a foveal or parafoveal macular hole. In two of these eyes, a thin transparent preretinal membrane was evident in the area of the staphyloma.

$\mathrm{RD}$ developed in 3 of $298 \mathrm{CE}$ eyes (1.0\%) in which laser

Table 3. Type of Retinal Detachment (RD) According to Degree of Myopia

\begin{tabular}{|c|c|c|c|c|c|c|c|c|c|c|}
\hline \multirow[b]{2}{*}{ Group } & \multirow[b]{2}{*}{$\mathrm{N}$} & \multirow{2}{*}{$\begin{array}{l}\text { Degree of Myopia } \\
\text { (negative diopters) }\end{array}$} & \multicolumn{4}{|c|}{$\begin{array}{c}\text { Peripheral RD with or without } \\
\text { Macular Involvement }\end{array}$} & \multicolumn{4}{|c|}{ Posterior RD } \\
\hline & & & $N$ & $B R d-T$ & BRd-UT & $\overline{\mathrm{ARd}}$ & $\bar{N}$ & $B R d-T$ & BRd-UT & $\overline{A R d}$ \\
\hline \multicolumn{11}{|l|}{ Control eyes } \\
\hline A & 5 & $15-19$ & 5 & 0 & 2 & 3 & 0 & 0 & 0 & 0 \\
\hline B & 4 & $20-24$ & 3 & 0 & 1 & 2 & 1 & 1 & 0 & 0 \\
\hline C & 2 & $25-30$ & 0 & 0 & 0 & 0 & 2 & 2 & 0 & 0 \\
\hline Total & 11 & $21.0 \pm 3.8$ & 8 & 0 & 3 & 5 & 3 & 3 & 0 & 0 \\
\hline \multicolumn{11}{|c|}{ Cataract-subjected eyes } \\
\hline A & 23 & $15-19$ & 21 & 4 & 5 & 12 & 2 & 0 & 0 & 2 \\
\hline B & 37 & $20-24$ & 13 & 1 & 4 & 8 & 24 & 10 & 1 & 13 \\
\hline $\mathrm{C}$ & 14 & $25-30$ & 1 & 1 & 0 & 0 & 13 & 8 & 0 & 5 \\
\hline Total & 74 & $20.7 \pm 4.2$ & 35 & 6 & 9 & 20 & 39 & 18 & 1 & 20 \\
\hline
\end{tabular}

$\mathrm{N}=$ number of eyes with RD; BRd-T = baseline retinal degeneration laser treated; BRd-UT $=$ baseline retinal degeneration untreated; ARd $=$ baseline absence of peripheral retinal degeneration. 
Table 4. Presence of Posterior Staphyloma (PS) and Its Relation to the Type of Retinal Detachment (RD)

\begin{tabular}{|c|c|c|c|c|c|c|c|}
\hline Group & $\mathrm{N}$ & $\mathrm{N}$ of PS & $\mathrm{N}$ of RD & $\begin{array}{l}\text { RD in } \\
\text { Eyes with } \\
\text { PS }\end{array}$ & $\begin{array}{c}\text { Peripheral RD (N } \\
\text { of eyes with PS/ } \\
\text { total eyes with } \\
\text { peripheral RD) }\end{array}$ & $\begin{array}{c}\text { Peripheral RD + MI } \\
\text { ( } \mathrm{N} \text { of eyes with PS/ } \\
\text { total eyes with } \\
\text { peripheral RD + } \\
\text { MI) }\end{array}$ & $\begin{array}{l}\text { Posterior RD }(\mathrm{N} \text { of } \\
\text { eyes with PS/total eyes } \\
\text { with posterior }(\mathrm{RD})\end{array}$ \\
\hline \multicolumn{8}{|l|}{ Control eyes } \\
\hline Group A & 431 & 36 & 5 & $0 / 36$ & $0 / 4(0.0 \%)$ & $0 / 4(0.0 \%)$ & 0/3 (0.0\%) \\
\hline Group B & 372 & 179 & 4 & $1 / 179$ & $0 / 4(0.0 \%)$ & $1 / 4(25 \%)$ & $0 / 3(0.0 \%)$ \\
\hline Group C & 127 & 127 & 2 & $2 / 127$ & $0 / 4(0.0 \%)$ & $0 / 4(0.0 \%)$ & $2 / 3(66.6 \%)$ \\
\hline Total & 930 & 342 & 11 & $3 / 342$ & $0 / 4(0.0 \%)$ & $1 / 4(25 \%)$ & $2 / 3(66.7 \%)$ \\
\hline \multicolumn{8}{|c|}{ Cataract subjected eyes } \\
\hline Group A & 431 & 48 & 23 & $6 / 48$ & $0 / 19(0.0 \%)$ & $5 / 16(31.25 \%)$ & $1 / 39(2.6 \%)$ \\
\hline Group B & 372 & 166 & 37 & $9 / 166$ & $0 / 19(0.0 \%)$ & $2 / 16(12.5 \%)$ & $7 / 39(17.9 \%)$ \\
\hline Group C & 127 & 127 & 14 & $14 / 127$ & $0 / 19(0.0 \%)$ & $1 / 16(6.25 \%)$ & $13 / 39(33.3 \%)$ \\
\hline Total & 930 & 341 & 74 & $29 / 341$ & $0 / 19(0.0 \%)$ & $8 / 16(50.0 \%)$ & $21 / 39(53.8 \%)$ \\
\hline
\end{tabular}

$\mathrm{MI}=$ macular involvement; $\mathrm{N}=$ number of eyes.

treatment for peripheral degenerations was carried out at baseline conditions; RD developed in 3 of 98 eyes $(3.1 \%)$ in which untreated peripheral degenerations were observed at baseline conditions and in 5 of 534 eyes $(0.9 \%)$ in which no peripheral degenerations were observed at baseline conditions. Details are presented in Table 5.

\section{CS Eyes}

In CS eyes, although the majority of cases of RD appeared in group B (eyes with myopia ranging from -20 to $-24 \mathrm{D}, 37$ of 431 CS eyes with RD [8.58\%]), considering the number of CS eyes in each group, a larger incidence of RD (14 of 127 CS eyes with RD [11.02\%]) was observed in group $\mathrm{C}$ (eyes with myopia ranging from -25 to -30 negative diopters).

The type of RD according to degree of myopia is described in Table 3.

In CS eyes, posterior RD was most frequently observed. This type of RD was found in 39 eyes $(52.7 \%, P>0.05$ vs. CE), whereas 19 eyes $(25.7 \%, P>0.05$ vs. $\mathrm{CE}$ ) showed peripheral RD, and 16 eyes $(21.6 \%, P>0.05$ vs. $\mathrm{CE})$ showed peripheral RD with macular involvement.

No posterior staphyloma was detected in the 19 CS eyes with peripheral RD; a posterior staphyloma was observed in 8 of the 16 (50\%) CS eyes with peripheral RD with macular involvement and in 21 of the $39(53.8 \%)$ CS eyes with posterior RD. Details are presented in Table 4.

\section{Peripheral RD in CS Eyes}

In 33 of 35 eyes with peripheral RD, there was PVD. In 2 eyes with peripheral RD, extensive vitreous liquefaction was found. The RD originated from peripheral retinal breaks on lattice areas in the superotemporal quadrant in 8 eyes, from multiple retinal breaks in 6 eyes, from large peripheral dialyses in 2 eyes, and from vitreoretinal tractions in the inferior quadrants in 18 eyes. Among the latter 18 eyes, 14 showed small retinal holes, whereas in 4 eyes, no retinal holes or tears were detected. In 1 eye, the RD originated from holes on lattice degeneration areas in the inferotemporal quadrant.

Table 5. Development of Retinal Detachment (RD) in Relation to the Absence or Presence of Peripheral Retinal Degeneration (laser-treated or untreated) at Baseline Evaluation

\begin{tabular}{lrr}
\hline & N in CEs & N in CS Eyes \\
\hline RD & & \\
RD in eyes with baseline absence of peripheral retinal degeneration & $5 / 534(0.9 \%)$ & $40 / 458(8.7 \%)^{*}$ \\
RD in eyes with baseline untreated retinal degeneration & $3 / 98(3.0 \%)$ & $10 / 86(11.6 \%)^{\dagger}$ \\
RD in eyes with baseline laser-treated retinal degeneration & $3 / 298(1.0 \%)$ & $24 / 386(6.21 \%)^{*}$ \\
RD total/total eyes enrolled & $11 / 930(1.2 \%)$ & $74 / 930(8.0 \%)^{*}$ \\
Peripheral RD with or without Macular involvement & $5 / 534(0.9 \%)$ & $3 / 98(3.0 \%)$ \\
RD in eyes with baseline absence of peripheral retinal degeneration & $0 / 298(0 \%)$ & $9 / 86(10 \%)^{*}$ \\
RD in eyes with baseline untreated retinal degeneration & $8 / 930(0.9 \%)$ & $6 / 386(1.5 \%)^{\dagger}$ \\
RD in eyes with baseline laser-treated retinal degeneration & & $35 / 930(3.8 \%)^{*}$ \\
RD total/total eyes enrolled & $0 / 534(0 \%)$ & $0 / 98(0 \%)$ \\
Posterior RD & $3 / 298(1.0 \%)$ & $20 / 458(4.4 \%)^{*}$ \\
RD in eyes with baseline absence of peripheral retinal degeneration & $3 / 930(0.3 \%)$ & $1 / 86(1.8 \%)^{\dagger}$ \\
RD in eyes with baseline untreated retinal degeneration & $18 / 386(4.7 \%)^{*}$ \\
RD in eyes with baseline laser-treated retinal degeneration & $39 / 930(4.2 \%)^{*}$
\end{tabular}

$\mathrm{N}=$ number of eyes; $\mathrm{CE}=$ control eye $\mathrm{CS}=$ cataract-subjected.

$* P<0.01$, chi-square with respect to CEs

${ }^{\dagger} P>0.01$, chi-square with respect to CEs. 
Yttrium-aluminum-garnet laser posterior capsulotomy had been previously performed in 18 of the 35 CS eyes $(51.4 \%)$ that developed peripheral RD.

\section{Posterior RD in CS Eyes}

In 32 of 39 eyes with posterior RD, there was extensive vitreous liquefaction, and in 26 eyes, a posterior vitreous schisis was clearly displayed in the form of a posterior vitreous pocket or two or more confluent lacunae, sometimes confined to the area of the staphyloma, sometimes greatly exceeding this area. In 7 of the 39 eyes, there was PVD.

Retinal detachment originated from a foveal or parafoveal hole in 14 eyes (in 9 eyes of this group, a thin and taut transparent membrane was observed in the area of the staphyloma); in 7 eyes, it originated from small paravascular retinal tears that in all cases were adherent to vitreous strands or veils; in 3 eyes, it originated from retinal breaks at the limit of the posterior pocket; and in 3 eyes, it originated from a break in the nasal ( 2 eyes) or inferior (1 eye) parapapillary area. In all cases, the breaks were adherent to a thin layer of posterior vitreous cortex. In 12 of 39 eyes, there was a flat posterior RD confined to the area of the posterior staphyloma. No retinal holes or tears were observed in this group. In 7 eyes of this group, a thin transparent preretinal membrane, at some points connected with the posterior inner retina, was observed.

Yttrium-aluminum-garnet laser posterior capsulotomy had been previously performed in 11 of the 39 CS eyes that developed posterior RD ( $28.2 \%, P<0.01$ vs. peripheral RD).

In CS eyes, RD developed in 24 of 386 eyes $(6.3 \%, P<0.01$ vs. CEs) in which laser treatment for peripheral degenerations was carried out at baseline conditions; RD developed in 10 of 86 eyes $(11.6 \%, P>0.01$ vs. CEs) in which untreated peripheral degenerations were observed at baseline conditions and in 40 out of 458 eyes $(8.7 \%, P<0.01)$ in which no peripheral degenerations were observed at baseline conditions. Details are presented in Table 5.

Of the $74 \mathrm{CS}$ eyes that developed RD, an IOL was implanted in 23 eyes, all belonging to group A (with myopia ranging between -15 and -19 D).

RD occurred in 29 out of the $318 \mathrm{CS}$ eyes $(9.1 \%)$ in which YAG laser posterior capsulotomy had been performed before the development of RD.

\section{Discussion}

It is known that both high myopia and cataract surgery may represent risk factors for the onset of RD. ${ }^{1-16}$ The aim of our study was to evaluate the risk for the onset of RD in highly myopic eyes after an uneventful cataract extraction.

In our subjects, we observed the development of RD after phacoemulsification in 74 of 930 eyes $(8.0 \%)$ and in 11 of 930 unoperated fellow eyes $(1.2 \%)$ during a follow-up period of 36 months. The highest incidence of RD (43.2\%) occurred between 25 and 36 months after surgery, and the largest incidence of RD (11.02\%) was observed in eyes with the highest degree of myopia (from -25 to $-30 \mathrm{D}$, axial length between 33.60 and $35.55 \mathrm{~mm}$ ).

Initially, 1024 subjects were enrolled in the study. Ninety-four subjects retired from the study during the first 6 months of follow-up, and therefore the final incidence of RD was calculated on the basis of the 930 subjects who had followed all complete postoperative examination at the preestablished times. We cannot determine whether the 94 subjects would have influenced the final incidence of RD.
Our results regarding the incidence of RD might seem in contrast to those reported by other authors.

In particular, several studies evaluated the onset of $\mathrm{RD}$ after phacoemulsification, reporting incidences of $0.8 \%, 7,15$ $0.9 \%,{ }^{12}$ and $1.17 \%{ }^{3}$ In these studies, the incidence of RD was evaluated over average follow-up periods of 32.2 months, ${ }^{7} 52$ months, ${ }^{15} 26.8$ months, ${ }^{12}$ and 40 months, ${ }^{3}$ and therefore with follow-up periods similar to that used in our study. Lyle and $\mathrm{Jin}^{12}$ reported the onset of RD in only 1 eye with an axial length of $27.36 \mathrm{~mm}$ out of 109 eyes (with a mean axial length of $28.7 \mathrm{~mm}$ ) that had undergone phacoemulsification. Alldredge et $\mathrm{al}^{13}$ reported no development of $\mathrm{RD}$ in 80 eyes that had undergone phacoemulsification, with a mean degree of myopia of $-10 \mathrm{D}$. However, the latter study evaluated subjects with a mean degree of myopia that was considerably lower than that of our subjects. In some studies, ${ }^{3,7,15}$ no correlation was reported between the onset of RD after cataract surgery and the degree of myopia. Nevertheless, several studies have suggested that the presence of high myopia increases the risk for RD after phacoemulsification. $3,7,13,15$

It is reported that YAG laser capsulotomy may represent a risk factor for the onset of RD in myopic eyes. In fact, a percentage of RD ranging from $0.17 \%$ to $3.2 \%$ after YAG laser capsulotomy was found, ${ }^{5,7,17-20}$ and this finding is further enhanced by our results in which RD was observed in a large percentage $(9.2 \%)$ of CS eyes that underwent YAG laser capsulotomy before the development of RD.

It has also been suggested that IOL implantation may prevent the onset of $\mathrm{RD}^{8}$ In our subjects, an IOL was implanted only with myopia between -15 and $-19 \mathrm{D}$, with an incidence of RD of $5.34 \%$, whereas in subjects without IOL, a greater incidence of RD was observed (groups B and C, $9.95 \%$ and $11.02 \%$, respectively). However, the role of IOL implantation cannot be determined from the present study because it was mostly carried out on group A eyes, and these eyes also had the lowest degree of myopia. Nevertheless, it is interesting to note that RD did not occur in any of the 44 group A eyes that received an IOL.

Similarly, a protective effect of prophylactic laser treatments on the onset of RD has been suggested. ${ }^{21}$

Prophylactic laser treatments were carried out preoperatively on a greater number of CS eyes with respect to CEs, and there were therefore different baseline conditions between the two groups regarding the retinal areas that required laser treatment. Indeed, prophylactic laser treatments were performed preoperatively in CS eyes with the intention of limiting the risk of developing retinal breaks and RD during or after surgery.

Nevertheless, no significant differences between lasertreated CS eyes and CEs were observed when peripheral RD developed, whereas a significant difference was observed when posterior RD developed (Table 5). Therefore, the observation of the anatomical features and locations of the RD that developed in laser-treated CS eyes and CEs (Table 5) suggests that the baseline variation of laser treatments in CS eyes and CEs did not play a role in the final incidence of RD observed in CS eyes and CEs.

In our CS eyes, we observed a greater incidence of peripheral $\mathrm{RD}$ in eyes with the absence of or untreated 
peripheral retinal degeneration, whereas the greatest incidence of posterior RD was observed in eyes with the absence of peripheral degeneration or in eyes that underwent laser treatment for peripheral retinal degeneration. All this leads us to believe in a possible prophylactic effect of laser treatment on the development of peripheral RD, although laser treatment cannot be considered to have a prophylactic effect on the development of posterior RD.

Regarding the time of onset of RD, in groups A and B, the distribution of RD cases is relatively balanced, with the highest incidence in the third year of follow-up and a reduction of cases in the second year; in group $\mathrm{C}$, there is a higher incidence of RD in the first and third year of follow-up and a significant reduction of cases in the second year.

Interesting considerations result from the analysis of the characteristics of RD in CS eyes and in their fellow eyes, especially with regard to those in which posterior RD occurred.

The type of RD seemed to be related to the degree of myopia: peripheral RD was more common in group A (eyes with a lower degree of myopia), whereas posterior RD was more common in group $\mathrm{C}$ (eyes with a higher degree of myopia).

In CS eyes, peripheral RD developed in those eyes in which no posterior staphyloma was observed; posterior RD developed mostly in eyes with the highest degree of myopia and in which a posterior staphyloma was detected.

In most eyes with a peripheral RD, with or without macular involvement, there was PVD. The RD was generally severe, and peripheral vitreoretinal tractions were in many cases evident, especially in RD originating from the inferior quadrants.

In most eyes with posterior $\mathrm{RD}$, there was a posterior vitreous schisis. The extension of RD was frequently limited to or slightly exceeding the limit of the posterior staphyloma.

RD was generally flat and clearly adherent to the vitreoretinal traction. Vitreous alterations were, in most cases, represented by a greater rigidity (condensation) of layers of the posterior vitreous cortex, which were focally or extensively adherent to the posterior retina in the form of a preretinal or epiretinal transparent membrane. These changes in the vitreous, in association with posterior vitreous schisis, can be considered a typical pathogenetic mechanism of posterior RD in highly myopic eyes in which a posterior staphyloma is associated with a posterior vitreous pocket. These eyes seem to be predisposed to posterior RD, and in our study, this predisposition was observed in both CS and CEs.

Therefore, the presence of vitreoretinal tractions and posterior staphyloma could be considered as additional risk factors for the development of posterior RD in highly myopic eyes subjected to cataract surgery.

To conclude, our study showed an incidence of RD that was significantly higher in eyes that underwent cataract surgery compared with the control group at 36 months of follow-up. RD, even after successful reparative surgery, was a substantial cause of serious visual loss in eyes in which the macular region was detached. Our study was limited to 36 months because many subjects wished to proceed with fellow eye surgery, and therefore, the incidence after longer follow-up remains unknown. Additional limitations of our study include IOL implantation and imbalances in a number of subjects with prophylactic peripheral retinal treatment, which was carried out in some eyes. Nevertheless, despite these limitations, cataract surgery seems to be a significant risk factor for RD in highly myopic eyes, even after uncomplicated phacoemulsification.

\section{References}

1. Goldberg MF. Clear lens extraction for axial myopia. An appraisal. Ophthalmology 1987;94:571-82.

2. Clayman HM, Jaffe NS, Light DS, et al. Intraocular lenses, axial length, and retinal detachment. Am J Ophthalmol 1981; 92:778-80.

3. Javitt JC, Vitale S, Canner JK, et al. National outcomes of cataract extraction I. Retinal detachment after inpatient surgery. Ophthalmology 1991;98:895-902.

4. Smith PW, Stark WJ, Maumenee AE, et al. Retinal detachment after extracapsular cataract extraction with posterior chamber intraocular lens. Ophthalmology 1987;94:495-504.

5. Tielsch JM, Legro MW, Cassard SD, et al. Risk factors for retinal detachment after cataract surgery. A population-based case-control study. Ophthalmology 1996;103:1537-45.

6. Wollensak J, Zeisberg B, Pham Duy T. Netzhautablosung nach Implantation einer Hinterkammerlinse [Retinal detachment following implantation of a posterior chamber lens]. Klin Monatsbl Augenheilkd 1988;192:1-5.

7. Coonan P, Fung WE, Webster RG Jr, et al. The incidence of retinal detachment following extracapsular cataract extraction. A ten-year study. Ophthalmology 1985;92:1096-101.

8. Lindstrom RL. Retinal detachment in axial myopia. Dev Ophthalmol 1987; 14:37-41.

9. Jacobi FK, Hessemer V. Pseudophakic retinal detachment in high axial myopia. J Cataract Refract Surg 1997;23:1095-102.

10. Percival SP. Redefinition of high myopia: the relationship of axial length measurement to myopic pathology and its relevance to cataract surgery. Dev Ophthalmol 1987;14:42-6.

11. Ogawa A, Tanaka M. The relationship between refractive errors and retinal detachment-analysis of 1,166 retinal detachment cases. Jpn J Ophthalmol 1988;32:310-5.

12. Lyle WA, Jin GJ. Phacoemulsification with intraocular lens implantation in high myopia. J Cataract Refract Surg 1996; 22:238-42.

13. Alldredge CD, Elkins B, Alldredge OC Jr. Retinal detachment following phacoemulsification in highly myopic cataract patients. J Cataract Refract Surg 1998;24:777-80.

14. Powell SK, Olson RJ. Incidence of retinal detachment after cataract surgery and neodymium: YAG laser capsulotomy. J Cataract Refract Surg 1995;21:132-5.

15. Olsen GM, Olson RJ. Prospective study of cataract surgery, capsulotomy, and retinal detachment. J Cataract Refract Surg 1995;21:136-9.

16. Jaffe NS, Clayman HM, Jaffe MS. Retinal detachment in myopic eyes after intracapsular and extracapsular cataract extraction. Am J Ophthalmol 1984;97:48-52.

17. Kraff MC, Sanders DR. Incidence of retinal detachment following posterior chamber intraocular lens surgery. J Cataract Refract Surg 1990;16:477-80.

18. Nissen KR, Fuchs J, Goldschmidt E, et al. Retinal detachment after cataract extraction in myopic eyes. J Cataract Refract Surg 1998;24:772-6. 


\section{Ripandelli et al - Cataract Surgery as a Risk Factor for Retinal Detachment}

19. Koch DD, Liu JF, Gill EP, Parke DW II. Axial myopia increases the risk of retinal complications after neodymiumYAG laser posterior capsulotomy. Arch Ophthalmol 1989; 107:986-90.

20. Fritch CD. Risk of retinal detachment in myopic eyes after intraocular lens implantation: a 7 year study. J Cataract Refract Surg 1998;24:1357-60.

21. Wilkinson CP, Rice TA. Prevention of retinal detachment. In: Wilkinson CP, Rice TA, eds. Michels Retinal Detachment. 2nd ed. St. Louis: CV Mosby; 1997;1081-113. 